\title{
Evaluation of Bioelectrical Impedance Spectroscopy for the Assessment of Extracellular Body Water
}

\author{
Sören Weyer, Lisa Röthlingshöfer, Marian Walter, Steffen Leonhardt \\ Philips Chair for Medical Information Technology, Helmholtz Institute for Biomedical Engineering, RWTH Aachen \\ University, Pauwelsstrasse 20, 52074 Aachen, Germany
}

Corresponding author: weyer@hia.rwth-aachen.de

\begin{abstract}
This study evaluates bioelectrical impedance spectroscopy (BIS) measurements to detect body fluid status. The multifrequency impedance measurements were performed in five female pigs. Animals were connected to an extracorporeal membrane oxygenation device during a lung disease experiment and fluid balance was recorded. Every 15 min the amount of fluid infusion and the weight of the urine drainage bag was recorded. From the fluid intake and output, the fluid balance was calculated. These data were compared with values calculated from a mathematical model, based on the extracellular tissue resistance and the Hanai Mixture theory. The extracellular tissue resistance was also measured with BIS. These experimental results strongly support the feasibility and clinical value of BIS for in vivo assessment of the hydration status.
\end{abstract}

Keywords: Bioelectrical impedance spectroscopy, fluid status, body composition, Hanai Mixture theory.

\section{Introduction}

Maintenance of a constant volume and stable composition of body fluids is essential for homeostasis. Several of the most common and serious problems in clinical medicine are due to abnormalities in the constancy of body fluid levels. Total body water is about $60 \%$ of body weight. This implies that in an (average) adult person weighing $70 \mathrm{~kg}$, total body water is about 42 liters. Total body fluid is distributed mainly between two compartments: the extracellular fluid consisting of plasma and interstitial fluid, and the intracellular fluid. Two thirds of the fluid is inside the cells and is called intracellular fluid. The remaining body fluid is located outside the cells and is called extracellular fluid. The extracellular fluid is mainly divided into interstitial fluid and blood plasma.

Edema is a condition that causes too much fluid to accumulate in the body. Any tissue or organ can be affected but the feet and ankles are the most affected; this is known as peripheral edema. Other common types of edema affect the lungs, brain and eyes [10]. The extracellular edemas (which occur most frequently) are generally caused by an abnormal leakage of fluid from plasma to the interstitial spaces across the capillaries, or by the failure of the lymphatic return from the interstitium back into the blood [10].

Due to the important role of body fluids, it is an important to accurately assess fluid status and its distribution. The gold standard for measurement of body water is isotope dilution; however, this method is only appropriate in a research setting. Other methods, like dual-energy X-ray absorptiometry, are costly and impractical for continuous monitoring [1]. A promising alternative for continuous monitoring fluid status is bioelectrical impedance spectroscopy (BIS). In BIS, detection of body hydration is based on the significant impact of body water on the electrical impedance.

\section{Bioimpedance}

BIS allows measurement of electrical body impedance at various frequencies, generally ranging from $5 \mathrm{kHz}$ to $1 \mathrm{MHz}$. The frequency-dependent impedance is related to the different electrical properties of body tissue. Biological tissue is often divided into three regions of dispersion: $\alpha, \beta$, and $\gamma$.

The $\alpha$ dispersion region is in the low frequencies, generally $<1 \mathrm{kHz}$ and mostly at frequencies $<10 \mathrm{~Hz}$. In this region impedance changes of only 1-2\% occur, mainly due to surface conductance, ion gates and cell membranes. The $\beta$ dispersion region is generally between $10 \mathrm{kHz}$ and $50 \mathrm{kHz}$ for muscular tissue and is mostly based on the phenomenon of the cell membrane. The two major contributing factors to $\beta$ dispersion are the shorting out of the membrane capacitances, and the rotational relaxation of biomacromolecules [8]. The $\gamma$ dispersion region is usually at very high frequencies. This dispersion is caused by the sub-cellular components of the tissue and by the water relaxation of the tissue. The frequency of the $\beta$ dispersion lies within the range which commercial BIS devices measure; therefore, this is explained in detail. The extracellular water content and intracellular 


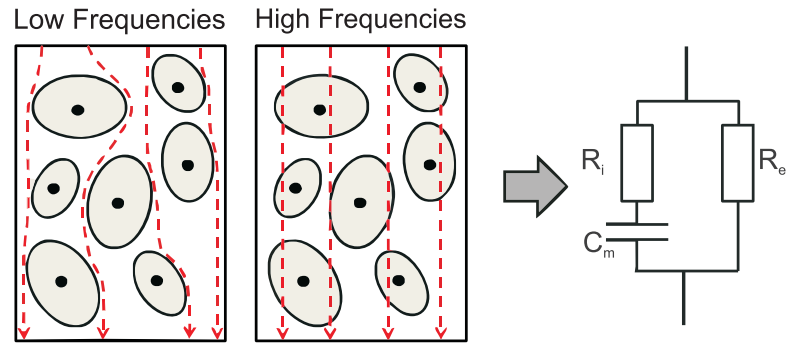

Figure 1: Low and high frequency current flow through the body. (Modified from [2].)

water content are mainly electrically resistive entities, whereas the cellular membrane (due to its lipid layer) has an isolating (capacitive) nature. Due to the conductive behavior of body fluid, the bio-electrical impedance is related to the fluid volume and its distribution [12]. Therefore, the impedance of tissue is strongly dependent on the frequency [2]. At low frequencies the extracellular fluid will conduct the current because cell membranes and tissue interfaces act as capacitors. Therefore, the current passes through the extracellular fluid and does not penetrate the cell membrane. At higher frequencies, this capacitive property is lost and the current is conducted by both types of fluid (see Fig. 1) [4, 2]. These physical characteristics are used to extrapolate the resistance of extracellular and intracellular fluid, which are related to fluid volume. Typical BIS measurements result in a semi-circular arc in the complex impedance plane (Fig. 3). Figure 1 also shows the electrical equivalent circuit which can be deduced from this behavior. This circuit and its associated equation is known as the Cole-Cole Model [3].

To calculate the resistance of intracellular and extracellular body water, the Cole-Cole model is used. As mentioned above, the Cole model represents the tissue using extracellular resistance $\left(R_{e}\right)$, an intracellular resistance $\left(R_{i}\right)$ and a capacitor (cell membrane capacitance $C_{m}$ ). The model was extended to include a heuristic factor $(\alpha)$ representing the presence of different tissues parallel with specific time constants [14]. Based on this model the impedance can be expressed by [13]:

$$
\begin{aligned}
Z(j \omega) & =R_{\infty}+\frac{R_{0}-R_{\infty}}{1+(j \omega \tau)^{\alpha}} e^{-j \omega T_{D}}, \\
\text { with: } R_{e} & =R_{0} \\
R_{i} & =\frac{R_{e} R_{\infty}}{R_{e}-R_{\infty}} \\
\tau & =\left(R_{i}+R_{e}\right) C_{m} .
\end{aligned}
$$

The values of the electrical model $R_{e}, R_{i}$ and $C_{m}$ can be found using the body impedance at the frequencies $\omega=0\left(R_{0}\right)$ and $\omega=\infty\left(R_{\infty}\right)$ and solving the equation for the parallel circuit. Due to the technical setup of this study, only frequencies between $5 \mathrm{kHz}$ and $1 \mathrm{MHz}$ are used. Thus, in practice, curve fitting methods are needed to calculate the parameters of the Cole-Cole model [2].

\section{Physiological Modelling}

Several physiological models have been proposed over the last decade. The simplest ones are the BIA Standard Model [5], the model known as $0 / \infty \mathrm{kHz}$ parallel model [9], the $50 \mathrm{kHz}$ parallel model [5, 9] and the $5 / 500-\mathrm{kHz}$ parallel model [9]. The main disadvantage of these models is their inaccuracy. The constant has to be suitable for each specific subject measured. In practice, because this is very laborious and not always possible, the calculations are only rough approximations. A more accurate model for the calculations, used in most commercial devices, is the Hanai suspension model $[11,6]$. In this model, the body is approximated by means of several conductive cylinders in series which represent the limbs and torso. All these cylinders are filled with a suspension containing nonconductive elements embedded in a conductive medium (extracellular fluid). Knowing the specific resistivity of the conductive medium, allows to calculate the partial volumes (conductive and nonconductive). According to the Hanais theory, the specific resistivity $\rho_{a}$ of a suspension of nonconductive spheres in a conductive medium is greater than the specific resistivity of the medium $\rho_{0}$ and depends on the fractional volume of the nonconductive spheres. This apparent resistivity is related to the conducting suspending medium $\rho_{0}$ and the volume fraction $c$ (dimensionless) of non-conducting spheres by $[11,6]$

$$
\rho_{a}=\frac{\rho_{0}}{(1-c)^{3 / 2}} .
$$

By substituting (2) with the formula for the resistance of a cylinder, which is

$$
R=\frac{\rho l}{A}=\frac{\rho l^{2}}{V},
$$

it is possible to calculate a cylinder filled with a suspension of nonconductive elements embedded in a conductive medium with the following equation:

$$
R=\frac{\rho_{0} H^{2}}{V(1-c)^{3 / 2}},
$$

where the $H$ has been substituted by the height and $V$ by the volume of the cylinder. Approximating the torso as a cylinder filled with a suspension of nonconductive elements in a conductive electrolyte, the nonconductive partial fraction $c$ can be defined as:

$$
c=1-\frac{V_{E C W}}{V_{B}},
$$


where $V_{B}$ constitutes the volume of the body and $V_{E C W}$ the volume of the extracellular fluid. Using (4) and (5), the body resistance can be rewritten as:

$$
R=\frac{\rho_{E C W} H^{2} V_{B}^{3 / 2}}{V_{B} V_{E C W}^{3 / 2}} .
$$

With (6), the extracellular fluid can be estimated as in $[13]$ :

$$
V_{E C W}=\frac{1}{1000}\left(\frac{\rho_{E C W}^{2}}{D_{B}}\right)^{\frac{1}{3}}\left(\frac{H^{2} \sqrt{W}}{R_{e}}\right)^{\frac{2}{3}}
$$

Here $W$ is the weight in $\mathrm{kg}$ and $H$ is the height in cm. $D_{B}$ is the body density, generally $1050 \mathrm{~kg} / \mathrm{m}^{3}$ for biological tissues. $\rho_{E C W}$ is the specific resistivity of the extracellular water and averages $40 \Omega \mathrm{cm}$ [16]. Thus, it is possible to predict the extracellular volume as long as the physiological parameters are known.

\section{Experimental setup}

The present study was realized as a sub-experiment during an extracorporeal membrane oxygenation (ECMO) trial in pigs.

In a typical extracorporeal oxygenation system, blood is collected from the body through largediameter cannulas placed in the femoral veins. From there, a blood pump transports the blood to the oxygenator and back into the body. In the present experiment, five female pigs (weight from 60 to 80 kilograms) were positioned supine, intubated and mechanically ventilated in volume-controlled mode. A double-lumen catheter was percutaneously inserted into the femoral veins, as it is standard in ECMO. The venous blood was drained by a blood pump. Once the blood passed through the ECMO circuit, it returned through a cannula which was inserted in the right internal jugular vein, and is then pushed forward so that the tip lies just above the right atrium. Body temperature of the animals was maintained by varying the temperature of a heating pad underneath the animal. The bladder was catheterized and an infusion started.

The ECMO circuit requires a high blood flow from the venous system. In order to avoid vasoconstriction in the femoral vein, a large volume of the fluid must be syringed. The infusion consisted of Ringer's solution (as required), a saline solution, or a hydroxyethyl starch solution. The amount of infusion supplied was measured every $15 \mathrm{~min}$ using the liquid level scale on the infusion bottle. The volume of urine was measured by weighing the urine drainage bag in order to evaluate the fluid balance of the animal. At the time of recording the fluid level, a BIS measurement was also performed. A Xitron Hydra 4200 system was

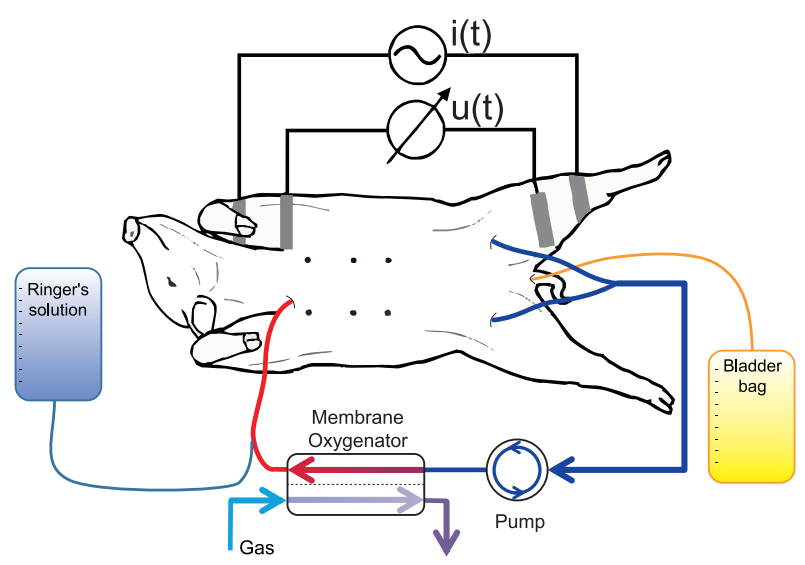

Figure 2: Sketch showing the position of the electrodes on the animal.

used to measure the bioimpedance at several frequencies. The device measures at 50 frequencies between $5 \mathrm{kHz}$ and $1000 \mathrm{kHz}$ and uses an excitation current in the range $50 \mu \mathrm{A}$ to $700 \mu \mathrm{A}$. The system is designed for a tetra-polar arrangement of electrodes. One pair of electrodes passes a small alternating current into the body and the other pair measures the resulting drop in voltage. The impedance is the ratio of voltage to current. This four-electrode technique reduces the effects of skin impedance $[7,15]$. Each measurement was repeated at least ten times to minimize measurement error due to movement artefacts caused by breathing, using an arithmetical averaging. In this setup, a current electrode and an adhesive measurement electrode were placed on the shaved skin of the anterior leg and the posterior leg of the pig (see Fig. 2); these electrodes were fixed with adhesive tape.

\section{Results}

Figure 3 shows the results of measurements in one pig. As expected, body impedance decreased with an increase in infused volume.

The bioelectrical parameters of each pig were estimated by fitting these curves to the Cole-Cole model (described by (1)) with the aim to relate its parameters to the physiological characteristics of the tissue. With the help of fitting algorithms, it is possible to determine the Cole-Cole model parameters $R_{e}, R_{i}, \alpha$ and $C_{m}$ from each curve.

In the present case, the extracellular fluid is particularly interesting since Ringer's solution is used to replace a deficit of extracellular fluid because it closely resembles physiological body fluid in terms of electrolyte concentration and osmolality. Hence, the extracellular fluid volume increases significantly after infusion of Ringer's lactate whereas the intracellular fluid volume remains constant. Thus, with the fitted $R_{e}$ values and the weight and height of the pigs it 


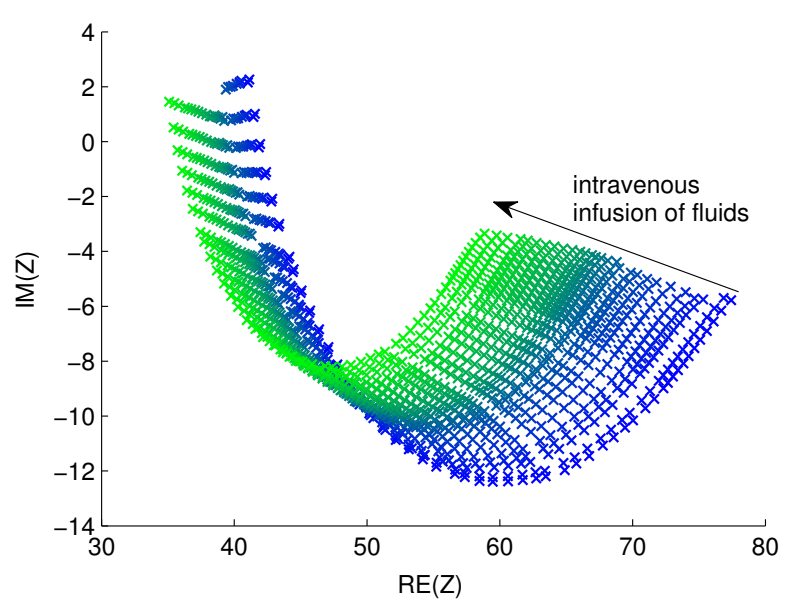

Figure 3: Plot of BIS measurement on the complex impedance plane, the fading color indicates the increase of liquid.

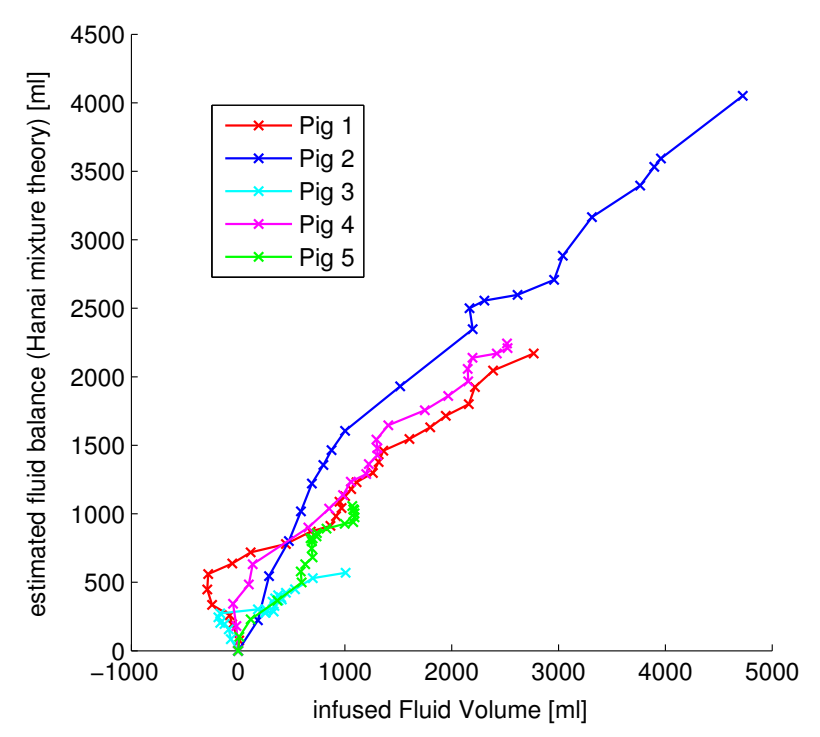

Figure 4: Estimation of fluid balance, calculated with the Hanai model, above the infused fluid volume.

is possible to calculate the extracellular fluid volume with (7).

Figure 4 shows the results of calculations with the Hanai model as a function of the change in fluid balance. To differentiate between the five pigs, different colors are used. Each measurement in each animal experiment corresponds to a plot in Fig. 4. As the fluid balance could only be recorded after catheterization of the animal in the operating room, the amount of liquid absorbed before the intervention could not be monitored and is unknown; in some cases a negative balance is observed at the start of the experiment. The amount of fluid supplied was mainly determined by the blood flow requirements of the ECMO. In addition, some pigs developed renal failure during the experiment and could no longer excrete urine. For

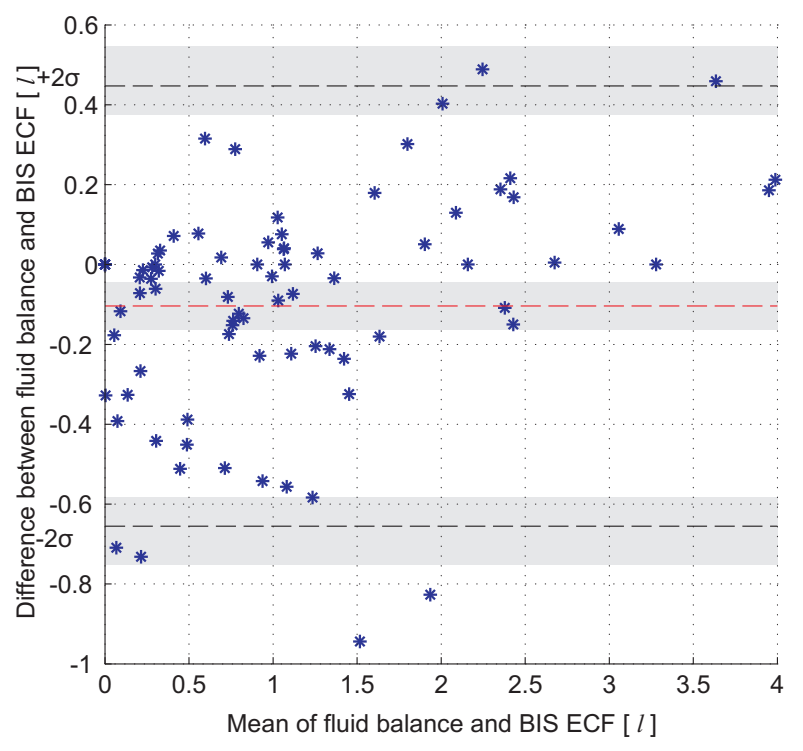

Figure 5: Bland-Altman plots illustrating the smallest and greatest difference between the changes in extracellular body fluid (ECF) estimated by BIS and the logged fluid balance plotted against the mean of the two methods.

this reason, the data sets do not cover the same range of fluid in all cases.

The Bland-Altman plots (Fig. 5) illustrate the differences between the BIS predicted fluid volume.

\section{Conclusion}

This study demonstrates that BIS can be used to continuously estimate extracellular water content. The limits of agreement (average difference \pm 1.96 standard deviation of the difference) are $0.45 \ell$ and $-0.65 \ell$. The mean difference between both methods is $-100 \mathrm{ml}$. This deviation may be due to evaporation related to breathing or sweat, and to the unknown fluid conditions before conducting the experiment. Initially, it seems as though the limits of agreement are large; however, the Bland-Altman plots show that in the physiological fluid range $(<1.5 \ell)$ the limits of agreement are $20 \%$ smaller. However, with a high fluid infusion the approximation of equation (7) loses its validity. BIS measurements are safe, non-invasive, portable, low cost, simple and provide instant results. The estimated fluid balance, calculated from BIS measurements, shows a strong correlation with the water balance. Furthermore, BIS is sensitive enough to detect fluid compartment differences and changes in fluid compartment volumes due to an acute intravenous administration of fluid. Therefore, BIS seems highly feasible to monitor water content in humans. 


\section{Acknowledgements}

The project has been selected under the operational programme co-financed by the European Regional Development Fund (ERDF) Objective 2 "Regional Competitiveness and Employment" 2007-2013), North Rhine-Westphalia (Germany).

\section{References}

[1] Carlina V. Albanese, Evelyn Diessel, Harry K. Genant. Clinical applications of body composition measurements using dxa. Journal of clinical densitometry : the official journal of the International Society for Clinical Densitometry 6(2):75-85, 2003.

[2] L. Beckmann, S. Hahne, G. Medrano, et al. Monitoring change of body fluids during physical exercise using bioimpedance spectroscopy. Annual International Conference of the IEEE Engineering in Medicine and Biology Society IEEE Engineering in Medicine and Biology Society Conference 2009:4465-4468, 2009.

[3] Kenneth Stewart Cole. Membranes, ions, and impulses. University of California Press, 1968.

[4] P. L. Cox-Reijven, P. B. Soeters. Validation of bio-impedance spectroscopy: effects of degree of obesity and ways of calculating volumes from measured resistance values. International journal of obesity and related metabolic disorders : journal of the International Association for the Study of Obesity 24(3):271-280, 2000.

[5] K. J. Ellis. Human body composition: in vivo methods. Physiological reviews 80(2):649-680, 2000 .

[6] M. Fenech, M. Maasrani, M. Y. Jaffrin. Fluid volumes determination by impedance spectroscopy and hematocrit monitoring: application to pediatric hemodialysis. Artificial organs 25(2):89-98, 2001.

[7] K. R. Foster, H. C. Lukaski. Whole-body impedance-what does it measure? The American journal of clinical nutrition 64(3 Suppl):388-396, 1996.
[8] Sverre Grimnes, Ørjan Grøttem Martinsen. Bioimpedance and bioelectricity basic. Academic Press, San Diego, second edition edn., 2000.

[9] R. Gudivaka, D. A. Schoeller, R. F. Kushner, M. J. Bolt. Single- and multifrequency models for bioelectrical impedance analysis of body water compartments. Journal of applied physiology (Bethesda, Md : 1985) 87(3):1087-1096, 1999.

[10] Arthur Clifton Guyton, John Edward Hall. Textbook of medical physiology. Elsevier Saunders, Philadelphia and PA, 11th edn., 2006.

[11] Tetsuya Hanai, Naokazu Koizumi, Rempei Gotoh. Dielectric properties of emulsions. KolloidZeitschrift \& Zeitschrift für Polymere 184(2):143148, 1962.

[12] E. C. Hoffer, C. K. Meador, D. C. Simpson. Correlation of whole-body impedance with total body water volume. Journal of applied physiology $27(4): 531-534,1969$.

[13] A. de Lorenzo, A. Andreoli, J. Matthie, P. Withers. Predicting body cell mass with bioimpedance by using theoretical methods: a technological review. Journal of applied physiology (Bethesda, Md : 1985) 82(5):1542-1558, 1997.

[14] Ulrich Moissl, Peter Wabel, Steffen Leonhardt, Rolf Isermann. Modellbasierte Analyse von Bioimpedanz-Verfahren (Model-based analysis of bioimpedance methods). at - Automatisierungstechnik 52(6-2004):270-279, 2004.

[15] J. Rosell, J. Colominas, P. Riu, et al. Skin impedance from $1 \mathrm{~Hz}$ to $1 \mathrm{MHz}$. IEEE Transactions on Biomedical Engineering 35(8):649-651, 1988.

[16] Xitron Technologies Inc. San Diego Californien, U.S.A. Hydra 4200 Bioimpedance Spectroscopy Operating Manual, 2007. 